\title{
Some extensions of Cartan-Behnke-Stein's theorem
}

\author{
By
}

Joji KAJIWARA

\section{Introduction}

Oka [15] proved that any domain of holomorphy in $C^{n}$ is a Cousin-I domain, that is, a domain in which any additive Cousin's distribution has a solution. Conversely Cartan [5] had stated that any Cousin-I domain in $C^{2}$ is a domain of holomorphy and Behnke-Stein [2] gave its proof. From Cartan $[6] C^{3}-\{(0,0,0)\}$ is a Cousin-I domain which is not a domain of holomorphy and from Thullen $[18] C^{2}-\{(0,0)\}$ is not a domain of holomorphy but a Cousin-II domain, that is, a domain in which any multiple Cousin's distribution has a solution. These two facts suggest that Cartan-Behnke-Stein's theorem can not be generalized for Cousin-I domain in $C^{n}(n \geqq 3)$ and for Cousin-II domain in $C^{n}(n \geqq 2)$ directly. The main purpose of the present paper is to extend Cartan-Behnke-Stein's theorem in the following form:

Let $L$ be an abelian complex Lie group and $\mathfrak{A}_{L}$ be the sheaf of all germs of holomorphic mappings in $L$. A domain $(D, \varphi)$ over $C^{2}$ with $\mathrm{H}^{1}\left(D, \mathfrak{2}_{L}\right)=0$ is a domain of holomorphy. If $D$ is a domain in $C^{n}$ with continuous boundary such that $\mathrm{H}^{1}\left(D \cap P, \mathfrak{A}_{L}\right)$ $=0$ for any simply connected and relatively compact polycylinder $P$ in $C^{n}, D$ is a domain of holomorphy. Moreover these two results hold also for $L=\mathrm{GL}(p, C)$.

Received April 28, 1966.

Communicated by S. Nakano.

Dedicated to Professor Wasao Sibagaki on his sixtieth birthday 
$\S 1$. Relation between domains of holomorphy and domains $D$ with ${ }^{*} \mathrm{H}^{1}\left(D, \mathfrak{A}_{L}\right)=0$.

Lemma. 1. Let $\mathfrak{F}$ be a sheaf of groups over a topological space $X$ and $\mathfrak{U}$ be an open covering of $X$. Then the canonical mapping $\mathrm{H}^{1}(\mathfrak{U}, \mathfrak{F}) \rightarrow \mathrm{H}^{1}(X, \mathfrak{F})$ is injective.

Proof. Suppose we have two cocycles $\left\{f_{i j}\right\},\left\{g_{i j}\right\} \in Z^{1}(\mathfrak{H}, \mathfrak{F})$ and suppose that there exist an open covering $\mathfrak{B}=\left\{V_{k} ; k \in K\right\}$, a cocycle $\left\{f_{k}\right\} \in \mathrm{C}^{0}(\mathfrak{S}, \mathfrak{F})$ and a mapping $\rho: K \rightarrow I$ satisfying the following conditions :

(1) $V_{k} \subset U_{\rho(k)}$, for any $k \in K$.

(2) $f_{k}^{-1} f_{\rho(k l)} f_{l}=g_{\rho(k l)}$ in any $V_{k} \cap V_{l} \neq \phi$ where $\rho(k l)=\rho(k) \rho(l)$.

(3) $\rho$ is surjective.

For any $i \in I$ we shall consider a fixed $k \in K$ with $\rho(k)=i$. For any $x \in U_{i}$ there exists $l \in K$ such that $x \in V_{l} \in \mathfrak{B}$. We put $\rho(l)=j$. If we put

$$
F_{i}(x)=f_{i j}(x) f_{l}(x)\left(g_{i j}(x)\right)^{-1}
$$

in $U_{i} \cap V_{l},\left\{F_{i}\right\} \in \mathrm{C}^{0}(\mathfrak{M}, \mathfrak{F})$ is well-defined and satisfies

$$
F_{\imath}^{-1} f_{i j} F_{j}=g_{i j}
$$

in $U_{i} \cap U_{j} \neq \phi$.

Lemma 2. Let $L$ be an abelian complex Lie group and $L_{e}$ be the connected component of $L$ containing the neutral element $e$. Then for a connected complex manifold $D$ the canonical mapping $\mathrm{H}^{1}\left(D, \mathfrak{A}_{L_{e}}\right) \rightarrow \mathrm{H}^{1}\left(D, \mathfrak{A}_{L}\right)$ is injective.

Proof. Let $\mathfrak{U}=\left\{U_{i} ; i \in I\right\}$ be an open covering of $D$ such that each $U_{i} \in \mathfrak{U}$ is connected. Suppose that a cocycle $\left\{f_{i j}\right\} \in Z^{1}\left(\mathfrak{U}, \mathfrak{A}_{\boldsymbol{L}_{i}}\right)$ is a coboundary of $\left\{f_{i}\right\} \in \mathrm{C}^{0}\left(\mathfrak{U}, \mathfrak{A}_{L}\right)$.

We take a fixed $i_{0} \in I$ and a fixed $x_{0} \in U_{i_{0}}$. If we put

$$
g_{i}(x)=f_{i}(x)\left(f_{i_{0}}\left(x_{0}\right)\right)^{-1}
$$

in $U_{i},\left\{f_{i j}\right\} \in Z^{1}\left(\mathfrak{U}, \mathfrak{A}_{L_{e}}\right)$ is a coboundary of $\left\{g_{i}\right\} \in \mathrm{C}^{0}\left(\mathfrak{U}, \mathfrak{A}_{L_{e}}\right)$.

If there exists a local biholomorphic mapping $\varphi$ of a complex manifold $D$ in a complex manifold $M,(D, \phi)$ is called an open 
set over $M$. Moreover, if $D$ is a connected complex manifold, $(D, \varphi)$ is called a domain over $M$. Let $\left(D_{1}, \varphi_{1}\right)$ and $\left(D_{2}, \varphi_{2}\right)$ be open sets over $M$. A holomorphic mapping $\lambda$ of $D_{1}$ in $D_{2}$ is called a mapping of $\left(D_{1}, \varphi_{1}\right)$ in $\left(D_{2}, \varphi_{2}\right)$ if $\varphi_{1}=\varphi_{2} \circ \lambda$. A complex manifold with vanishing fundamental group is called simply connected. Let $(D, \varphi)$ be a domain over $M$ and $\Im$ be a family of holomorphic functions on $D$. A triple $(\lambda, \tilde{D}, \tilde{\varphi})$, or shortly a pair $(\bar{D}, \tilde{\varphi})$, is called an envelope of holomorphy of $(D, \varphi)$ with respect to $\widetilde{\&}$ if the following conditions are satisfied:

(1) $\lambda$ is a mapping of $(D, \varphi)$ in $(\tilde{D}, \tilde{\varphi})$.

(2) For any $f \in \mathfrak{F}$ there exists a holomorphic function $\tilde{f}$ on $\tilde{D}$ with $f=\tilde{f} \circ \lambda$. (In this case $\tilde{f}$ is called an analytic continuation of $f$ to $(\lambda, \tilde{D}, \tilde{\varphi}))$.

(3) For any $\left(\lambda^{\prime}, D^{\prime}, \varphi^{\prime}\right)$ satisfying (1) and (2) (such is called an analytic completion of $(D, \varphi)$ with respect to $\widetilde{F})$ there exists a mapping $\psi$ of $\left(D^{\prime}, \varphi^{\prime}\right)$ in $(\tilde{D}, \tilde{\Phi})$ such that $(\psi, \tilde{D}, \tilde{\varphi})$ is an analytic completion of $\left(D^{\prime}, \varphi^{\prime}\right)$ with respect to the family of all analytic continuations of functions of $\mathfrak{F}$.

Cartan [7] proved the unique existence of such envelope of holomorphy. Especially if $\mathfrak{₹}$ consists of only one holomorphic function $f$ on $D$, the envelope of holomorphy of $(D, \varphi)$ with respect to $\mathfrak{F}$ is called a domain of holomorphy of $f$. A domain over $M$ which is a domain of holomorphy of a holomorphic function on a domain over $M$ is called shortly a domain of holomorphy. Moreover if $\mathfrak{F}$ is the family of all holomorphic functions on $D$, the envelope of holomorphy of $(D, \varphi)$ with respect to $₹$ is called shortly the envelope of holomorphy of $(D, \varphi)$.

Let $(D, \varphi)$ be a domain over $M$. A triple $\left(D^{\prime}, \varphi^{\prime}, \lambda\right)$ is called a covering domain of $(D, \varphi)$ if the following condition is satisfied:

$\lambda$ is a mapping of $\left(D^{\prime}, \varphi^{\prime}\right)$ on $(D, \varphi)$ and for any point $x$ of $D$ there exists a neighbourhood $U$ of $x$ such that $\lambda$ maps each 
connected component of $\lambda^{-1}(U)$ biholomorphically onto $U$.

Sometimes we also say that $\left(D^{\prime}, \lambda\right)$ is a covering domain of $D$. A covering domain $\left(D^{\sharp}, \phi^{\sharp}, \lambda\right)$ of $(D, \varphi)$ satisfying the following condition is called a universal covering domain of $(D, \varphi)$ :

For any covering domain $\left(D^{\prime}, \varphi^{\prime}, \lambda^{\prime}\right)$ of $(D, \varphi)$ there exists a mapping $\mu$ such that $\left(D^{\sharp}, \phi^{\sharp}, \mu\right)$ is a covering domain of $\left(D^{\prime}, \phi^{\prime}\right)$.

The universal covering domain of a domain over $M$ exists uniquely and a covering domain $\left(D^{\prime}, \varphi^{\prime}, \lambda\right)$ of $(D, \varphi)$ is a universal covering domain of $(D, \varphi)$ if and only if $D^{\prime}$ is simply connected.

Now let $(D, \varphi)$ be a domain over $M$ and $\left(D^{\sharp}, \varphi^{\sharp}, \lambda\right)$ be its universal covering domain. $\lambda$ induces canonically a mapping $\lambda^{*}: \mathrm{H}^{1}\left(D, \mathfrak{A}_{L}\right) \rightarrow \mathrm{H}^{1}\left(D^{*}, \mathfrak{A}_{L}\right)$ for a complex Lie group $L$.

$$
{ }^{*} \mathrm{H}^{1}\left(D, \mathfrak{A}_{L}\right)=\lambda^{*}\left(\mathrm{H}^{1}\left(D, \mathfrak{\Re}_{L}\right)\right)
$$

is a subgroup of $\mathrm{H}^{1}\left(D^{\sharp}, \mathfrak{A}_{L}\right)$ if $L$ is abelian. For $\alpha \in \mathrm{H}^{1}\left(D, \mathfrak{A}_{L}\right)$ we put

$$
{ }^{\sharp} \alpha=\lambda^{*}(\alpha) \in \mathrm{H}^{1}\left(D^{\sharp}, \mathfrak{A}_{\mathrm{L}}\right)
$$

and use these notations frequently hereafter.

Lemma 3. Let $L$ be a p-dimensional abelian complex Lie group, $(D, \varphi)$ be a domain over $C^{n}$ with ${ }^{*} \mathrm{H}^{1}\left(D, \mathfrak{A}_{L}\right)=0$ and $\left(D^{\sharp}\right.$, $\left.\varphi^{\sharp}, \lambda\right)$ be the universal covering domain of $(D, \varphi)$. Then for any $(n-1)$-dimensional analytic plane $H$ and for any holomorphic function $u$ on $\phi^{-1}(H)$, there exists a holomorphic function $F$ on $D^{\sharp}$ such that $F=u \circ \lambda$ in $\phi^{\sharp-1}(H)=\lambda^{-1}\left(\phi^{-1}(H)\right)$.

Proof. From Lemma 2 we may assume that $L$ is connected. As well known, there exists a homomorphism $\chi$ of the additive group $C^{p}$ on $L$ such that $\left(C^{p}, \chi\right)$ is a covering domain of $L$. In this case the kernel $N$ of $\chi$ is a discrete subgroup of $C^{p}$ isomorphic with the fundamental group of $L$. There exists a holomorphic function $u^{\prime}$ on a neighbouhood $V$ of $\varphi^{-1}(H)$ such that $u^{\prime}=u$ in $\varphi^{-1}(H)$. If we put $W=D-\phi^{-1}(H),\{V, W\}$ is an open covering of $D$. We consider 


$$
\chi\left(\frac{u^{\prime}}{z_{1} \circ \varphi}, 0, \cdots, 0\right) \in H^{0}\left(V \cap W, \mathfrak{A}_{\mathbf{L}}\right) .
$$

As ${ }^{\sharp} \mathrm{H}^{1}\left(D, \mathfrak{R}_{L}\right)=0$, from Lemma 1 there exist $A \in \mathrm{H}^{0}\left(\lambda^{-1}(V), \mathfrak{A}_{L}\right)$ and $B \in \mathrm{H}^{0}\left(\lambda^{-1}(W), \mathfrak{R}_{L}\right)$ such that

$$
\chi\left(\frac{u^{\prime} \circ \lambda}{z_{1} \circ \varphi^{\#}}, 0, \cdots, 0\right)=A B^{-1}
$$

in $\lambda^{-1}(V \cap W)$. Since $\left(C^{p}, \chi\right)$ is a covering domain of $L$, there exist, respectively, vector-valued holomorphic functions $a=\left(a_{1}\right.$, $\left.a_{2}, \cdots, a_{p}\right)$ and $b=\left(b_{1}, b_{2}, \cdots, b_{x}\right)$ on a simply connected subdomain of $\lambda^{-1}(V \cap W)$ with $A=\chi(a)$ and $B=\chi(b)$. Since $\chi$ is a homomorphism, for a suitable choice of $a$ and $b$ there holds

$$
\left(z_{1} \circ \varphi^{\sharp}\right) a=\left(u^{\prime} \circ \lambda, 0, \cdots, 0\right)+\left(z_{1} \circ \varphi^{\sharp}\right) b .
$$

Moreover $a$ and $b$ can be analytically continued along any curve in $\lambda^{-1}(V)$ and $\lambda^{-1}(W)$ respectively. If we denote their analytic continuations by the same symbols $a$ and $b$, for any simply connected subdomain $E$ of $\lambda^{-1}(V \cap W)$ there exists a constant $c(E) \in N$ such that

$$
\left(z_{1} \circ \varphi^{\sharp}\right) a=\left(u^{\prime} \circ \varphi^{\sharp}, 0, \cdots, 0\right)+\left(z_{1} \circ \varphi^{\sharp}\right) b+\left(z_{1} \circ \varphi^{\sharp}\right) c(E)
$$

in $E$. This means that $\left(z_{1} \circ \varphi^{\sharp}\right) a_{1}$ can be analytically continued along any curve in $D^{\sharp}$. Since $D^{\sharp}$ is simply connected, from the principle of monodromy this gives a holomorphic function $F$ on $D^{\sharp}$. Again from the above equation we have

$$
F=u \circ \lambda
$$

in $\varphi^{\sharp-1}(H)$.

Proposition 1. Let $(D, \varphi)$ be a domain over $C^{2}$. If ${ }^{\sharp} \mathrm{H}^{1}(D$, $\left.\mathfrak{A}_{L}\right)=0$ for an abelian complex Lie group $L,(D, \varphi)$ is a domain of holomorphy.

Proof. The set $E$ of all points of $\partial D$ which is a boundary point of $\varphi^{-1}(H)$ for some analytic plane $H$ is dense in $\partial D$. Since $\varphi^{-1}(H)$ is holomorphically convex from Behnke-Stein [3], there exists a holomorphic function $u$ on $\phi^{-1}(H)$ which is un- 
bounded at $x$ for any boundary point $x$ of $\varphi^{-1}(H)$. From Lemma 3 there exists a holomorphic function $F$ on $D$ which is unbounded at boundary points of $\partial D^{\ddagger}$ over $x$. Since $E$ is dense in $\partial D$, each boundary point of $D^{\sharp}$ has the frontier property in the sense of Bochner-Martin [4]. There exists a holomorphic function $G$ on $D^{*}$ which is unbounded at each point of $\partial D^{\sharp}$. Since $\left(D^{\sharp}, \varphi^{\sharp}\right)$ is a covering domain of the domain $\left(D^{\prime}, \phi^{\prime}\right)$ of holomorphy of $G,\left(D^{\prime}, \varphi^{\prime}\right)$ is a covering domain of $(D, \varphi)$. Making use of Oka [16] or Stein [17] we can prove that a domain over $C^{n}$ is a domain of holomorphy if and only if its covering domain is a domain of holomorphy. Hence $(D, \varphi)$ is a domain of holomorphy.

Corollary to Proposition 1 . Let $L$ be a p-dimensional connected abelian complex Lie group, $\pi(L)$ be its fundamental group and $(D, \varphi)$ be a domain over $C^{2}$. Then $\mathrm{H}^{1}\left(D, \mathfrak{A}_{L}\right)=0$ if and only if $(D, \varphi)$ is a domain of holomorphy with $\mathrm{H}^{2}(D, \pi(L))=0$.

Proof. If $\mathrm{H}^{1}\left(D, \mathfrak{A}_{L}\right)=0,(D, \varphi)$ is a domain of holomorphy from Proposition 1. Since $\mathrm{H}^{1}\left(D, \mathfrak{S}^{p}\right)=\mathbb{H}^{2}\left(D, \mathfrak{O}^{p}\right)=0$ in the canonical exact sequence

$$
\mathrm{H}^{1}\left(D, \bigcirc^{p}\right) \rightarrow \mathrm{H}^{1}\left(D, \mathfrak{R}_{L}\right) \rightarrow \mathrm{H}^{2}(D, \pi(L)) \rightarrow \mathrm{H}^{2}\left(D, \mathfrak{O}_{p}\right)
$$

where $\bigcirc^{p}=\mathfrak{A}_{\sigma^{p}}$, we have $\mathrm{H}^{2}(D, \pi(L))=0$. Conversely if $(D, \varphi)$ is a domain of holomorphy with $\mathrm{H}^{2}(D, \pi(L))=0$, we have $\mathrm{H}^{1}\left(D, \mathfrak{A}_{L}\right)=0$ from the above exact sequence.

Making use of Lemma 3 we can prove the following proposition by induction with respect to $n$ similarly to Prosition 1 .

Proposition 2. A domain $(D, \varphi)$ over $C^{n}$ which satisfies ${ }^{*} \mathrm{H}^{1}\left(\varphi^{-1}(H), \mathfrak{A}_{L}\right)=0$ for any analytic plane $H$ represented by $H=\left\{z ; z_{q_{1}}=a_{1}, z_{q_{2}}=a_{2}, \cdots, z_{q_{n-m}}=a_{n-m}\right\} \quad(2 \leqq m \leqq n)$ is a domain of holomorphy.

\section{§2. Approximation of holomorphic functions}

A collection $\widetilde{S}=\left\{\left(D_{n}, \varphi_{n}\right) ; \tau_{m}^{n}\right\}$ is called a monotonously increasing sequence of domains over a complex manifold $M$ if the 
following conditions are satisfied:

(1) Each $\left(D_{n}, \Phi_{n}\right)$ is a domain over $M$.

(2) Each $\tau_{m}^{n}(n \leqq m)$ is a mapping of $\left(D_{n}, \varphi_{n}\right)$ in $\left(D_{m}, \varphi_{m}\right)$ with $\tau_{l}^{n}=\tau_{l}^{m} \circ \tau_{m}^{n}$ for $n \leqq m \leqq l$.

A triple $\left(\tau_{n}, D, \varphi\right)$, or shortly a pair $(D, \mathscr{P})$, is called a limit of $\subseteq$ if the following conditions are satisfied:

(1) $(D, \varphi)$ is a domain over $M$.

(2) Each $\tau_{n}$ is a mapping of $\left(D_{n}, \varphi_{n}\right)$ in $(D, \varphi)$ with $\tau_{n}=\tau_{m} \circ \tau_{m}^{n}$ for $n \leqq m$.

(3) If $\left(\tau_{n}^{\prime}, D^{\prime}, \varphi^{\prime}\right)$ satisfies (1) and (2), there exists a mapping $\psi$ of $(D, \varphi)$ in $\left(D^{\prime}, \varphi^{\prime}\right)$ with $\tau_{n}^{\prime}=\psi \circ \tau_{n}$ for any $n$.

As we stated in [11] the limit of $\subseteq$ exists uniquely and has the following property :

Lemma 4. Let $\left(\tau_{n}, D, \varphi\right)$ be the limit of a monotonously increasing sequence $\left\{\left(D_{n}, \varphi_{n}\right) ; \tau_{m}^{n}\right\}$ of domains over a complex manifold $M$. Then for any compact set $K$ in $D$ there exist an integer $m$ and a compact set $K^{\prime}$ in $D_{n}$ such that $\tau_{m}$ maps $K^{\prime}$ biholomorphically on $K$.

Let $\tau$ be a mapping of a domain $\left(D_{1}, \varphi_{1}\right)$ over a Stein manifold $S$ in a domain $\left(D_{2}, \varphi_{2}\right)$ over $S,\left(\lambda_{1}, \tilde{D}_{1}, \tilde{\varphi}_{1}\right)$ and $\left(\lambda_{2}, \tilde{D}_{2}, \tilde{\varphi}_{2}\right)$ be, respectively, the envelopes of holomorphy of $\left(D_{1}, \varphi_{1}\right)$ and $\left(D_{2}, \varphi_{2}\right)$. As we remarked in [11], there exists a mapping $\tilde{\tau}$ of $\left(\tilde{D}_{1}, \tilde{\varphi}_{1}\right)$ in $\left(\tilde{D}_{2}, \tilde{\varphi}_{2}\right)$ with $\tilde{\tau} \circ \lambda_{1}=\lambda_{2} \circ \tilde{\tau} . \quad \tilde{\tau}$ is called an analytic continuation of $\tau$ to $\left(\lambda, \tilde{D}_{1}, \tilde{\tau}_{1}\right)$.

We have the following Lemma as we remarked in [11].

Lemma 5. Let $\left(\tau_{n}, D, \varphi\right)$ be a limit of a monotonously increasing sequence $\left\{\left(D_{n}, \varphi_{n}\right) ; \tau_{m}^{n}\right\}$ of domains over a Stein manifold $S,\left(\lambda_{n}, \tilde{D}_{n}, \tilde{\varphi}_{n}\right)$ and $(\lambda, \tilde{D}, \tilde{\varphi})$ be, respectively, the envelopes of holomorphy of $\left(D_{n}, \varphi_{n}\right)$ and $(D, \varphi), \tilde{\tau}_{m}^{n}$ and $\tilde{\tau}_{n}$ be, respectively, the analytic continuations of $\tau_{m}^{n}$ and $\tau_{n}$ to $\left(\lambda_{n}, \tilde{D}_{n}, \tilde{\varphi}_{n}\right)$. Then $\left\{\left(\tilde{D}_{n}\right.\right.$, $\left.\left.\tilde{\rho}_{n}\right) ; \tilde{\tau}_{m}^{n}\right\}$ is a monotonously increasing sequence of domains over $S$ and $\left(\tilde{\tau}_{n}, \tilde{D}, \tilde{\varphi}\right)$ is its limit.

Let $\tau$ be a mapping of a domain $\left(D_{1}, \varphi_{1}\right)$ over a complex 
manifold $M$ in a domain $\left(D_{2}, \varphi_{2}\right)$ over $M,\left(D_{1}^{\sharp}, \varphi_{1}^{\sharp}, \lambda_{1}\right)$ and $\left(D_{2}^{\sharp}\right.$, $\left.\varphi_{2}^{*}, \lambda_{2}\right)$ be, respectively, the universal covering domains of $\left(D_{1}, \varphi_{1}\right)$ and $\left(D_{2}, \varphi_{2}\right)$. Then there exists a mapping $\tau^{\#}$ of $\left(D_{1}^{\sharp}, \varphi_{1}^{\sharp}\right)$ in $\left(D_{2}^{*}, \phi_{2}^{*}\right)$ with $\tau \circ \lambda_{1}=\lambda_{2} \circ \tau^{*}$. $\quad \tau^{*}$ is called a mapping of $\left(D_{1}^{*}, \varphi_{1}^{*}\right)$ in $\left(D_{2}^{*}, \phi_{2}^{*}\right)$ associated to the mapping $\tau$. We have the following Lemma.

Lemma 6. Let $\left(\tau_{n}, D, \varphi\right)$ be the limit of a monotonously increasing sequence $\left\{\left(D_{n}, \varphi_{n}\right) ; \tau_{m}^{n}\right\}$ of domains over a complex mainfold $M,\left(D_{n}^{\sharp}, \varphi_{n}^{\sharp}, \lambda_{n}\right)$ and $\left(D^{\sharp}, \phi^{\sharp}, \lambda\right)$ be, respectively, the universal covering domains of $\left(D_{n}, \varphi_{n}\right)$ and $(D, \varphi), \tau_{m}^{n \#}$ and $\tau_{n}^{\#}$ be, respectively, the mappings of $\left(D_{n}^{\sharp}, \varphi_{n}^{\sharp}\right)$ in $\left(D_{m}^{\sharp}, \phi_{m}^{\sharp}\right)$ and $\left(D^{\sharp}, \varphi^{\sharp}\right)$ associated to the mappings $\tau_{m}^{n}$ and $\tau_{n}$. Then $\left\{\left(D_{n}^{\sharp}, \varphi_{n}^{\sharp}\right) ; \tau_{m}^{n \sharp}\right\}$ is a monotonously increasing sequence of domains over $M$ and $\left(\tau_{n}^{\sharp}\right.$, $\left.D^{\sharp}, \varphi^{\sharp}\right)$ is its limit.

Under these preperations we shall give the following Lemma.

Lemma 7. Let $\left\{\left(D_{n}, \varphi_{n}\right), \tau_{m}^{n}\right\}$ be a monotonously increasing sequence of domains over a Stein manifold $S$ and $\left(\tau_{n}, D_{n}, \varphi_{n}\right)$ be its limit. Let $\left\{K_{n}\right\}$ be a sequence of compact subsets of $D$ such that $K_{n} \subset K_{n+1}$ and $D=\bigcup_{n=1}^{\infty} K_{n}$. Then there exists a subsequence $\left\{\nu_{n}\right\}$ of $\{1,2,3, \cdots\}$ with the following properties:

(1) There exists a compact subset $K_{n}^{\prime}$ of $D_{\nu_{n}}$ such that the restriction $\tau_{\nu_{n}} \mid K_{n}^{\prime}$ of $\tau_{\nu_{n}}$ to $K_{n}$ is a biholomorphic mapping of $K_{n}^{\prime}$ onto $K_{n}$.

(2) For any $f_{n} \in \mathrm{H}_{0}\left(D_{\nu_{n}}, \mathfrak{O}\right)$ and $\varepsilon_{n}>0$ there exists $F_{n} \in \mathrm{H}^{0}$ $(D, \bigcirc)$ such that $\left|F_{n} \circ \tau_{\nu_{n}}-f_{n}\right|<\varepsilon_{n}$ in $K_{\nu_{n}}^{\prime}$.

Proof. Let $\left(\lambda_{n}, D_{n}, \varphi_{n}\right)$ and $(\lambda, D, \varphi)$ be, respectively, the envelopes of holomorphy of $\left(D_{n}, \varphi_{n}\right)$ and $(D, \varphi), \tilde{\tau}_{m}^{n}$ and $\tilde{\tau}_{n}$ be, respectively, the analytic continuations of $\tau_{m}^{n}$ and $\tau_{n}$ to $\left(\lambda_{n}, \tilde{D}_{n}\right.$, $\left.\tilde{\varphi}_{n}\right)$ and $(\lambda, \tilde{D}, \tilde{\varphi})$. From Lemma $5\left\{\left(\check{D}_{n}, \tilde{\varphi}_{n}\right) ; \tau_{m}^{n}\right\}$ is the limit of a monotonously increasing sequence of domains over $S$ and $\left(\tilde{\tau}_{n}\right.$, $\tilde{D}, \tilde{\varphi})$ is its limit. Since $\tilde{D}$ is holomorphically convex, there exists a sequence of analytic polycylinders $P_{n}$ in $\tilde{D}$ such that 


$$
\lambda\left(K_{n}\right) \subset P_{n} \subset P_{n+1}
$$

for any $n \geqq 1$. Since $\left(\tau_{n}, D, \varphi\right)$ and $\left(\tilde{\tau}_{n}, \tilde{D}, \tilde{\varphi}\right)$ are, respectively, limits of $\left\{\left(D_{n}, \varphi_{n}\right) ; \tau_{m}^{n}\right\}$ and $\left\{\left(\tilde{D}_{n}, \tilde{\varphi}_{n}\right) ; \tilde{\tau}_{m}^{n}\right\}$, from Lemma 4 there exists a subsequence $\left\{\nu_{n}\right\}$ of $\{1,2,3, \cdots\}$ such that $\tau_{\nu_{n}}$ and $\tilde{\tau}_{\nu_{n}}$ map, respectively, a compact subset $K_{n}^{\prime}$ of $D_{\nu_{n}}$ and an open subset $P_{n}^{\prime}$ of $\tilde{D}_{\nu_{n}}$ onto $K_{n}$ and $P_{n}$ and that

$$
\tau_{\nu_{n+1}}^{\nu_{n}}\left(P_{n}^{\prime}\right) \subset P_{n+1}^{\prime}, \lambda_{\nu_{n}}\left(K_{n}^{\prime}\right) \subset P_{n}^{\prime} .
$$

Now let $f_{n}$ be any holomorphic function on $D_{\iota_{n}}$. Since $\left(\lambda_{\nu_{n}}, \tilde{D}_{\nu_{n}}, \tilde{\varphi}_{\nu_{n}}\right)$ is the envelope of holomorphy of $\left(D_{\nu_{n}}, \varphi_{\nu_{n}}\right)$, there exists $\tilde{f}_{n} \in \mathrm{H}^{0}\left(\tilde{D}_{\nu_{n}}, \mathfrak{D}\right)$ such that $\tilde{f}_{n} \circ \lambda_{\nu_{n}}=f_{n}$.

Then

$$
\tilde{f}_{n} \circ\left(\tilde{\tau}_{\nu_{n}} \mid P_{n}^{\prime}\right)^{-1} \in \mathrm{H}^{0}\left(P_{n}, \mathfrak{D}\right) \text {. }
$$

Since $P_{n}$ is holomorphically convex with respect to $\tilde{D}$, there exists $\tilde{F}_{n} \in \mathrm{H}^{0}(\tilde{D}, \mathfrak{D})$ such that

$$
\left|F_{n}-f_{n} \circ\left(\tilde{\tau}_{\nu_{n}} \mid P_{n}^{\prime}\right)^{-1}\right|<\varepsilon_{n}
$$

in $K_{n}$. Then $F_{n}=\tilde{F}_{n} \circ \lambda$ satisfies

$$
\left|F_{n} \circ \tau_{\nu_{n}}-f_{n}\right|<\varepsilon_{n}
$$

in $K_{n}^{\prime}$.

\section{$\S 3$. Limit of cohomology groups}

Let $\left(\tau_{n}, D, \varphi\right)$ be a limit of a monotonously increasing sequence $\left\{\left(D_{n}, \varphi_{n}\right) ; \tau_{m}^{n}\right\}$ of domains over a complex manifold $M$ and $\mathfrak{A}_{L}$ be the sheaf of all germs of holomorphic mappings in a complex Lie group $L . \quad \tau_{m}^{n}$ induces canonically the mapping $\pi_{n}^{m}: \mathrm{H}^{1}\left(D_{m}, \mathfrak{A}_{L}\right) \rightarrow \mathrm{H}^{1}\left(D_{n}, \mathfrak{A}_{L}\right)$ for $n \leqq m$ such that $\pi_{n}^{l}=\pi_{n}^{m} \circ \pi_{m}^{l}$ for $n \leqq m \leqq l$. Hence $\left\{\mathrm{H}^{1}\left(D_{n}, \mathfrak{A}_{L}\right) ; \pi_{n}^{m}\right\}$ forms an inverse system over a directed set $\{1,2,3, \cdots\}$. Its inverse limit is denoted by $\lim$ $\mathrm{H}^{1}\left(D_{n}, \mathfrak{A}_{L}\right)$. The canonical mappings $\pi_{n} ; \lim \mathrm{H}^{1}\left(D_{n}, \mathfrak{A}_{L}\right) \rightarrow$ $\mathrm{H}^{1}\left(D_{n}, \mathfrak{A}_{L}\right)$ and $\pi: \mathrm{H}^{1}\left(D, \mathfrak{A}_{L}\right) \rightarrow \lim \mathrm{H}^{1}\left(D_{n}, \mathfrak{A}_{L}\right)$ are also considered. If an element $\alpha$ of $\mathrm{H}^{1}\left(D, \mathfrak{A}_{L}\right)$ is the canonical image of an element 
of $\mathrm{B}^{1}\left(\mathfrak{U}, \mathfrak{A}_{\mathbb{L}}\right)$ for an open covering $\mathfrak{U}$ of $D$, we say that $\alpha=0$. We say that $\mathrm{H}^{1}\left(D, \mathfrak{A}_{L}\right)=0$ if $\alpha=0$ for any $\alpha \in \mathrm{H}^{1}\left(D, \mathfrak{A}_{L}\right)$. If $\alpha \in \lim \mathrm{H}^{1}\left(D_{n}, \mathfrak{A}_{L}\right)$ satisfies $\pi_{n}(\alpha)=0$ for any $n \geqq 1$, we say that $\alpha=0$. If $\pi(\alpha)=0$ implies $\alpha=0$ for $\alpha \in \mathrm{H}^{1}\left(D, \mathfrak{P}_{L}\right)$, we say that $\pi$ is quasi-injective. If $L$ is abelian and $\pi$ is quasi-injective, $\pi$ is injective. Let $L$ be a complex Lie group and $L_{a}$ be the connected component of $L$ containing $a$. For $a, b \in L$ the mapping $\tau$ defined by $\tau(x)=\mathrm{ba}^{-1} x$ for $x \in L$ maps $L_{a}$ biholomorphically onto $L_{b}$. Hence, if $\left(C^{p}, \chi\right)$ is a covering domain of $L_{e},\left(C^{p}, \chi_{a}\right)$ is a covering domain of $L_{a}$ for any $a \in L$, where $e$ is the neutral element of $L$ and $\chi_{a}(z)=a \chi(z)$ for $z \in C^{p}$. If $L$ is a $p$-dimensional abelian or soluble complex Lie group, $\left(C^{p}, \chi\right)$ is a covering domain of $L_{e}$ for suitable $\chi$.

Lemma 8. Let $\left(C^{p}, \chi\right)$ be a covering manifold of $L_{e}$ for $a$ complex Lie group $L,\left\{\left(D_{n}, \varphi_{n}\right) ; \tau_{m}^{n}\right\}$ be a monotonously increasing sequence of simply connected domains over a Stein manifold $S$ and $\left(\tau_{n}, D, \varphi\right)$ be its limit. Then the canonical mapping $\mathbb{H}^{1}\left(D, \mathfrak{A}_{L}\right)$ $\rightarrow \lim \mathrm{H}^{1}\left(D_{n}, \mathfrak{A}_{L}\right)$ is quasi-injective.

Proof. In this proof we shall denote a point of $C^{n}$ by a gothic type as $a$ and the inverse element of an element $a$ of the group $L$ by $a^{-1}$. If we put

$$
\chi_{a}(z)=a \chi(z)
$$

for $z \in C^{p},\left(C^{p}, \chi_{a}\right)$ is a covering domain of $L_{a}$ for $a \in L$. Let $\left\{K_{n}\right\}$ be a sequence of compact subsets of $D$ such that $K_{n}$ is contained in the open kernel of $K_{n+1}$ and $D=\bigcup_{n=1}^{\infty} K_{n}$. If we apply Lemma 7 to this $\left\{K_{n}\right\}$, we obtain a subsequence $\left\{\nu_{n}\right\}$ of $\{1,2,3, \cdots\}$ and a sequence $\left\{K_{n}^{\prime}\right\}$ satisfying the conditions (1) and (2) in Lemma 7. Without loss of generality we may suppose that $\nu_{n}=n$.

There exists $e \in C^{p}$ with $\chi(e)=e$ for the neutral element $e$ of $L$. We consider the Euclidean distance in $C^{p}$. Let $\mathbb{W}^{\prime}$ be an open sphere with centre $\mathbb{e}$ and semiradius $r$ such that $\chi$ 
maps $W^{\prime}$ biholomorphically onto an after neighbourhood $W^{\prime}$ of e. We put

$$
\begin{gathered}
\operatorname{dist}(a, b)=\operatorname{dist}\left(\left(\chi \mid W^{\prime}\right)^{-1} a,\left(\chi \mid W^{\prime}\right)^{-1} b\right) \quad\|a\|=\operatorname{dist}(e, a), \\
W=\left\{z ; \operatorname{dist}(e, z)<\frac{r}{2}\right\}, W=\chi(\mathbb{W})
\end{gathered}
$$

where $a, b \in W^{\prime}$ and $\chi \mid W^{\prime}$ is the restriction of $\chi$ to $W^{\prime}$. For any $\varepsilon>0$ there exists $\delta(\varepsilon)>0$ satisfying the following condition:

$0<\delta(\varepsilon)<\varepsilon . \quad$ If $a, b \in W$ satisfy $\|b\|<\delta(\varepsilon), a b \in W^{\prime}$ and $\operatorname{dist}(a$, $a b)<\varepsilon$.

From Lemma 1 it suffices to prove that $f_{i j} \in \mathrm{B}^{1}\left(\mathfrak{U}, \mathfrak{A}_{L}\right)$ for an open covering $\mathfrak{U}=\left\{U_{i} ; i \in I\right\}$ of $D$ if $\left\{f_{i j}^{\circ} \tau_{n}\right\} \in \mathrm{B}^{1}\left(\tau_{n}^{-1}(\mathfrak{H}), \mathfrak{A}_{L}\right)$ for $n \geqq 1$. The condition means that $\left\{f_{i j} \circ \tau_{n}\right\}$ satisfies

$$
f_{i j} \circ \tau_{n}=h^{\prime \prime}\left(h_{j}^{\prime \prime}\right)^{-1}
$$

in $\tau_{n}^{-1}\left(U_{l}\right) \cap \tau_{n}^{-1}\left(U_{j}\right)$ for a suitable $\left\{h^{n}\right\} \in \mathrm{C}^{0}\left(\tau_{n}^{-1}(U), \mathfrak{A}_{L}\right)$, where $\left\{\tau_{n}^{-1}(\mathfrak{M})=\tau_{n}^{-1}\left(U_{i}\right) ; i \in I\right\}$ an open covering of $D_{n}$. If we put

$$
f^{1}=\left(h^{1}\right)^{-1}\left(h^{2} \circ \tau_{2}^{1}\right)
$$

in $\tau_{1}^{-1}\left(U_{i}\right), f^{1} \in \mathrm{H}^{0}\left(D_{1}, \mathfrak{2}_{L}\right)$ is well-defined. Since $D_{1}$ is connected, $f^{1}\left(D_{1}\right) \subset L_{a_{1}}$ for some $a_{1} \in L$. We put $\chi_{1}=\chi_{a_{1}}$. Since $D_{1}$ is simply connected and $\left(C^{p}, \chi_{1}\right)$ is a universal covering domain of $L_{a_{1}}$, from the principle of monodromy there exists $\mathbb{F}^{1} \in \mathrm{H}^{0}\left(D_{1}\right.$, $\left.\mathfrak{S}^{p}\right)$ such that $f^{1}=\chi_{1} \circ \mathbb{H}^{1}$. From Lemma 7 there exists $\mathbb{H}^{1} \in$ $\mathrm{H}^{0}\left(D, \mathfrak{D}^{p}\right)$ such that the image of $K_{1}^{\prime}$ by the mapping $f^{1}\left(\chi_{1} \circ\right.$ $\left.H^{1} \circ \tau_{1}\right)^{-1}$ is contained in $W$ and

$$
\left\|f^{1}\left(X_{1} \circ H^{1} \circ \tau_{1}\right)^{-1}\right\|<\delta\left(r 2^{-3}\right)
$$

in $K_{1}^{\prime}$. If we put

$$
\left\{g_{i}^{1}\right\}=\left\{h_{l}^{1}\right\} \in \mathrm{C}^{0}\left(\tau_{2}^{-1}(\mathfrak{H}), \mathfrak{A}_{L}\right)
$$

and

$$
g_{\iota}^{2}=h_{\iota}^{2}\left(\chi_{1} \circ \mathbb{H}^{1} \circ \tau_{2}\right)^{-1}
$$

in $\tau_{2}^{-1}\left(U_{i}\right)$, the coboundary of $\left\{g_{\imath}^{2}\right\} \in \mathrm{C}^{0}\left(\tau_{2}^{-1}(\mathfrak{U}), \mathfrak{N}_{L}\right)$ is $\left\{f_{i j} \circ \tau_{2}\right\}$. If we put 


$$
g^{1,2}=\left(g_{\imath}^{1}\right)^{-1}\left(g_{\imath}^{2} \circ \tau_{2}^{1}\right)
$$

in $\tau_{1}^{-1}\left(U_{i}\right), g^{1,2} \in \mathrm{H}^{0}\left(D_{1}, \mathfrak{A}_{L}\right)$ is well-defined, the image of $K_{1}^{\prime}$ by the mapping $g^{1,2}$ is contained in $W$ and

$$
\left\|g^{1,2}\right\|<\delta\left(r 2^{-3}\right)
$$

in $K_{1}^{\prime}$. Suppose that there exist $\left\{g_{\imath}^{1}\right\} \in \mathrm{C}^{0}\left(\tau_{1}^{-1}(\mathfrak{U}), \mathfrak{A}_{L}\right),\left\{g_{\imath}^{2}\right\} \in$ $\mathrm{C}^{0}\left(\tau_{2}^{-1}(\mathfrak{U}), \mathfrak{A}_{L}\right), \cdots$ and $\left\{\mathfrak{g}_{\imath}^{m}\right\} \in \mathrm{C}^{0}\left(\tau_{m}^{-1}(\mathfrak{H}), \mathfrak{A}_{L}\right)$ satisfying the following conditions :

(1) The coboundary of $\left\{g_{\imath}^{n}\right\}$ is $\left\{f_{i j}^{\circ} \tau_{n}\right\}$ for $n$ with $1 \leqq n \leqq m$.

(2) If we put

$$
g^{n, l}=\left(g_{i}^{n}\right)^{-1}\left(g_{\imath}^{l} \circ \tau_{l}^{n}\right)
$$

in $\tau_{n}^{-1}\left(U_{i}\right)$ for any $1 \leqq n<l \leqq m, g^{n, l} \in \mathrm{H}^{0}\left(D_{n}, \mathfrak{A}_{t}\right)$ is well-defined, the image of $K_{n}^{\prime}$ by the mapping $g^{n, n+1}$ is contained in $W$ and

$$
\left\|g^{n, n+1}\right\|<\delta\left(r 2^{-n-2}\right)
$$

in $K_{n}^{\prime}$.

If we put

$$
f^{m}=\left(g_{i}^{m}\right)^{-1}\left(h^{m+1} \circ \tau_{m+1}^{m}\right)
$$

in $\tau_{m}^{-1}\left(U_{i}\right), f^{m} \in \mathrm{H}^{0}\left(D_{m}, \mathfrak{A}_{\Sigma}\right)$ is well-defined. Since $D_{m}$ is connected, there exists $a_{m} \in L$ such that $f^{m}\left(D_{m}\right) \subset L_{a_{m}}$. We put $\chi_{m}=\chi_{a_{m}}$. Since $D_{m}$ is simply connected and $\left(C^{p}, \chi_{m}\right)$ is a covering domain of $L_{a_{m}}$, there exists $\mathbb{F}^{m} \in \mathrm{H}^{0}\left(D_{m}, S^{p}\right)$ such that $f^{m}=\chi_{m} \circ \boldsymbol{F}^{m}$. From the condition (2) of Lemma $7 \boldsymbol{F}_{m}$ can be uniformly approximated in $K_{m}^{\prime}$ by functions in $\mathrm{H}^{0}\left(D, \bigcirc^{p}\right)$ so that the image of $K_{m}^{\prime}$ by the mapping $f^{m}\left(\chi_{m} \circ H^{m} \circ \tau_{m}\right)^{-1}$ is contained in $W$ and

$$
\left\|f^{m}\left(\chi_{m} \circ H^{m} \circ \tau_{m}\right)^{-1}\right\|<\delta\left(r 2^{-m-2}\right)
$$

in $K_{n}^{\prime}$. If we put

$$
g_{i}^{m+1}=h_{i}^{m+1}\left(\chi_{m} \circ H^{m} \circ \tau_{m+1}\right)^{-1}
$$

in $\tau_{m+1}^{-1}\left(U_{i}\right)$, the coboundary of $\left\{g_{\imath}^{m+1}\right\} \in \mathrm{C}^{0}\left(\tau_{m+1}^{-1}(\mathfrak{U}), \mathfrak{A}_{L}\right)$ is $\left\{f_{i j} \circ \tau_{m+1}\right\}$ $\in Z^{1}\left(\tau_{m+1}^{-1}(\mathfrak{U}), \mathfrak{A}_{L}\right)$. If we put

$$
g^{m, m+1}=\left(g_{\imath}^{m}\right)^{-1}\left(g_{\imath !}^{m+1} \circ \tau_{m+1}^{m}\right)
$$


in $\tau_{m}^{-1}\left(U_{i}\right), g^{m, m+1} \in \mathrm{H}^{0}\left(D_{m}, \mathfrak{N}_{\boldsymbol{L}}\right)$ is well-defined and the image of $K_{m}^{\prime}$ by the mapping $g^{m, m+1}$ is contained in $W$ and

$$
\left\|g^{m, m+1}\right\|<\delta\left(r 2^{-m-2}\right)
$$

in $K_{m}^{\prime}$. In this way we can construct $\left\{g_{\imath}^{m}\right\} \in \mathrm{C}^{0}\left(\tau_{m}^{-1}(\mathfrak{U}), \mathfrak{A}_{L}\right)$ satisfying the above conditions (1) and (2) inductively for $m=$ $1,2,3, \cdots$.

The image of $K_{n}^{\prime}$ by the mapping $g^{n, m}$ is contained in $W$,

$$
\| g^{n, m}||<r 2^{-n-1}
$$

in $K_{n}^{\prime}$ for $1 \leqq n \leqq m$ and

$$
\operatorname{dist}\left(g^{n, m}, g^{n, m+1}\right)<r 2^{-m-2}
$$

in $K_{n}^{\prime}$ for $n \leqq m$. Therefore the subsequence $\left\{(\chi \mid W)^{-1} g^{n, m}\right.$; $m=n, n+1, \cdots\}$ of $\mathrm{H}^{0}\left(D_{n}, \bigcirc^{p}\right)$ tends uniformly to $\mathbb{G}^{n} \in \mathrm{H}^{0}\left(\tau_{n}^{n-1}\right.$ $\left.\left(K_{n-1}^{\prime}\right), \mathfrak{S}^{p}\right)$ in $\tau_{n}^{n-1}\left(K_{n-1}^{\prime}\right)$ for $n=2,3,4, \cdots$. Since

$$
\operatorname{dist}\left(e, G^{n}\right)<r 2^{-n}
$$

in $\tau_{n}^{n-1}\left(K_{n-1}^{\prime}\right)$ for $n=2,3,4, \cdots, \quad\left\{g^{n, m} ; m=n, n+1, \cdots\right\}$ tends uniformly to

$$
g^{n}=\chi_{\circ} G^{n} \in H^{0}\left(\tau_{n}^{n-1}\left(K_{n-1}^{\prime}\right), \mathfrak{A}_{\mathbf{L}}\right)
$$

in $\tau_{n}^{n-1}\left(K_{n-1}^{\prime}\right)$. If we put

$$
f_{i}=\left(g_{i}^{n} g^{n}\right) \circ\left(\tau_{n} \mid K_{n}^{\prime}\right)^{-1}
$$

in $K_{n-1}$ for $n$ with $U_{i} \subset K_{n-1},\left\{f_{i}\right\} \in \mathrm{C}^{0}\left(\mathfrak{U}, \mathfrak{A}_{L}\right)$ is well-defined and its coboundary is the original cocycle $\left\{f_{i j}\right\} \in Z^{1}\left(\mathfrak{U}, \mathfrak{A}_{L}\right)$.

The above proof of Lemma 8 gives the following Corollary.

Corollary to Lemma 8. Let $(D, \varphi)$ be a limit of a monotonously increasing sequence $\left\{\left(D_{n}, \varphi_{n}\right)\right\}$ of domains over a Stein manifold. Then the canonical homomorphism $\mathrm{H}^{1}\left(D, \bigcirc^{p}\right) \rightarrow$ $\lim \mathrm{H}^{1}\left(D_{n}, \bigcirc^{p}\right)$ is injective for $p \geqq 1$.

Let $\left(\tau_{n}, D, \varphi\right)$ be a limit of a monotonously increasing sequence $\left\{\left(D_{n}, \varphi_{n}\right), \tau_{m}^{n}\right\}$ of domains over $M$. Let $\tau_{n}^{\sharp}:\left(D_{n}^{\sharp}, \varphi_{n}^{\sharp}\right) \rightarrow$ $\left(D^{\sharp}, \phi^{\sharp}\right)$ and $\tau_{m}^{n \sharp}:\left(D_{n}^{\sharp}, \phi_{n}^{\sharp}\right) \rightarrow\left(D_{m}^{\sharp}, \varphi_{m}^{\sharp}\right)$ be, respectively, the mappings 
associated to $\tau_{n}$ and $\tau_{m}^{n}$ for $1 \leqq n \leqq m$. Then $\left(\tau_{n}^{\sharp}, D^{\#}, \phi^{\sharp}\right)$ is a limit of a monotonously increasing sequence $\left\{\left(D_{n}^{\sharp}, \varphi_{n}^{\sharp}\right), \tau_{m}^{n \sharp}\right\}$ of domains over $M . \quad \tau_{n} \circ \lambda_{n}$ 's induce a canonical mapping $\pi^{*}: \mathbb{H}^{1}\left(D, \mathfrak{2}_{L}\right) \rightarrow$ $\lim \mathbb{H}^{1}\left(D_{n}^{\sharp}, \mathfrak{A}_{L}\right)$. Under these notations we have the following Lemma.

Lemma 9. Let $\left(C^{p}, \chi\right)$ be a covering domain of a connected component of a complex Lie group $L,\left\{\left(D_{n}, \varphi_{n}\right), \tau_{m}^{n}\right\}$ be a monotonously increasing sequence of domains over a Stein manifold $S$ and $\left(\tau_{n}, D, \varphi\right)$ be its limit. For any $\alpha \in \mathrm{H}^{1}\left(D, \mathfrak{A}_{L}\right)$ with ${ }^{*} \pi(\alpha)=0$, we have ${ }^{*} \alpha=0$.

Proof. From Lemma 4 it suffices to prove that any $\left\{f_{i j}\right\} \in$ $Z^{1}\left(\mathfrak{U}, \mathfrak{A}_{L}\right)$ for an open covering $\mathfrak{U}$ of $D$ with $\left\{f_{i j} \circ \tau_{n} \circ \lambda_{n}\right\} \in \mathrm{B}^{1}\left(\lambda_{n}^{-1}\right.$ $\left.\left.\tau_{n}^{-1}(\mathfrak{U})\right), \mathfrak{A}_{L}\right)(n \geqq 1)$ satisfies $\left\{f_{i j} \circ \lambda\right\} \in \mathrm{B}^{1}\left(\lambda^{-1}(\mathfrak{U}), \mathfrak{A}_{L}\right)$. Since $\tau_{n} \circ \lambda_{n}$ $=\lambda \circ \tau_{n}^{*}$ for any $n$,

$$
\left\{f_{i j} \circ \lambda \circ \tau_{n}^{\sharp}\right\} \in \mathrm{B}^{1}\left(\tau_{n}^{\sharp-1}\left(\lambda^{-1}(\mathfrak{U})\right), \mathfrak{A}_{x}\right)
$$

for any $n$. As each $D_{n}^{*}$ is simply connected, we have

$$
\left\{f_{i j} \circ \lambda\right\} \in \mathrm{B}^{1}\left(\lambda^{-1}(\mathfrak{U}), \mathfrak{A}_{\mathfrak{L}}\right)
$$

from Lemma 8.

As a corollary to Lemma 9 we have the following Proposition.

Proposition 3. Let $L$ be an abelian or soluble complex Lie group, $\left\{\left(D_{n}, \varphi_{n}\right)\right\}$ be a monotonously increasing sequence of domains over a Stein manifold and $(D, \varphi)$ be its limit. If $D$ is simply connected, the canonical mapping $\mathrm{H}^{1}\left(D, \mathfrak{A}_{\bar{L}}\right) \rightarrow \lim \mathrm{H}^{1}\left(D_{n}\right.$, $\mathfrak{A}_{L}$ ) is quasi-injective.

\section{§4. Cousinn-I and Cousin- $\mathbb{I I}$ distributions}

Let $L$ be an abelian or soluble complex $\mathbb{L i e}$ group and $D$ be a domain in a Stein manifold $S$. Let $\widetilde{\gamma}$ be a subset of $\mathrm{H}^{1}\left(D, \mathfrak{A}_{L}\right)$. For a subdomain $E$ of $D$ we consider the inclusion mapping $i_{E}: E \rightarrow D$. Let $\left(E^{*}, \lambda_{E}\right)$ be the universal covering domain of $E . \quad i_{E}$ and $i_{H} \circ \lambda_{E}$ induce canonically mappings 
$i_{E}^{*}: \mathrm{H}^{1}\left(D, \mathfrak{A}_{L}\right) \rightarrow \mathrm{H}^{1}\left(E, \mathfrak{A}_{L}\right)$ and $\lambda_{E}^{*} \circ i_{E}^{*}: \mathrm{H}^{1}\left(D, \mathfrak{A}_{L}\right) \rightarrow \mathrm{H}^{1}\left(E^{*}, \mathfrak{A}_{L}\right)$. For $\alpha \in \mathrm{H}^{1}\left(D, \mathfrak{A}_{L}\right)$ we put $\alpha \mid E=i_{E}^{*}(\alpha)$ and ${ }^{\sharp}(\alpha \mid E)=\lambda_{E}^{*}\left(i_{E}^{*}(\alpha)\right)$. We consider the set $E_{\widetilde{F}}$ of all subdomains $E$ of $D$ such that ${ }^{\sharp}(\alpha \mid E)=0$ for any $\alpha \in \mathfrak{F}$. Then $\left(E_{\mathfrak{F}}, \subset\right)$ forms a partially ordered set. Let $\mathfrak{\complement}=\left\{C_{t} ; t \in T\right\}$ be a totally ordered subset of $\left(E_{\Im}, \subset\right)$. We put

$$
C=\bigcup_{t \in T} C_{t} .
$$

Then $C$ is a subdomain of $D$. There exists a sequence $\left\{K_{n} ; n=1,2,3, \cdots\right\}$ of compact subsets of $C$ such that

$$
K_{n} \subset K_{n+1}, C=\bigcup_{n=1}^{\infty} K_{n} .
$$

Since $\mathfrak{C}$ is an open covering of a compact set $K_{1}$ and $(\mathfrak{C}, \subset)$ is totally ordered, there exists $C_{1} \in \mathbb{C}$ such that $\mathbb{K}_{1} \subset C_{\mu_{1}}$. Suppose that there exists $C_{\mu_{1}}, C_{\mu_{2}}, \cdots, C_{\mu_{n}} \in \mathfrak{C}$ such that

$$
C_{\mu_{1}} \subset C_{\mu_{2}} \subset \cdots \subset C_{\mu_{n}}, K_{m} \subset C_{\mu_{m}}(1 \leqq m \leqq n) .
$$

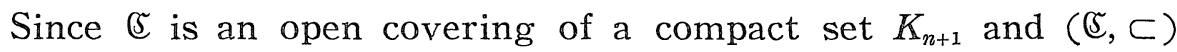
is totally ordered, there exists $C_{\mu_{n+1}}^{\prime} \in \mathbb{C}$ such that $K_{n+1} \subset C_{\mu_{n+1}}^{\prime}$. Then we have

$$
C_{\mu_{n+1}}=C_{\mu_{n}} \cup C_{p_{n+1}}^{\prime} \in \mathfrak{S}
$$

and

$$
C_{\mu_{n}} \subset C_{\mu_{n+1}}, K_{n+1} \subset C_{\mu_{n+1}} .
$$

Thus we have proved the existence of $\left\{C_{\mu_{n}} ; n=1,2,3, \cdots\right\} \subset \mathfrak{c}$ such that

$$
C_{\mu_{1}} \subset C_{\mu_{2}} \subset \cdots \subset C_{\prime_{n}} \subset \cdots, C=\bigcup_{n=1}^{\infty} C_{\mu_{n}} .
$$

Since $"\left(\alpha \mid C_{\mu_{n}}\right)=0$ for any $n$ and any $\alpha \in \mathfrak{F}$, we have $"(\alpha \mid C)=0$ for any $\alpha \in \mathfrak{F}$ from Lemma 9. Therefore $C \in E_{\mathscr{F}}$ and $C$ is an upper bound of ( 5 . From Zorn's Lemma there exists a maximal element $D_{\mathfrak{F}}$ of $\left(E_{\mathscr{F}}, \subset\right)$ which is an upper bound of $\mathfrak{c}$. 
A collection $\widetilde{C}=\left\{\left(m_{i}, U_{i}\right) ; i \in I\right\}$ of pairs of an open subset $U_{i}$ of $D$ and a meromorphic function $m_{i}$ in $U_{i}$ is called a CousinII distribution in $D$ if the following conditions are satisfied:

(1) $\mathfrak{U}=\left\{U_{i} ; i \in I\right\}$ is an open covering of $D$.

(2) $\rho(\mathfrak{C})=\left\{m_{i} m_{\jmath}^{-1}\right\} \in \mathbb{Z}^{1}\left(\mathfrak{U}, \mathfrak{O}^{*}\right)$ where $\mathfrak{O}^{*}=\mathfrak{A}_{\mathrm{GL}(1, c)}$.

Let $E$ be a subdomain of $D$ and $\left(E^{*}, \lambda\right)$ be the universal covering domain of $E$. A meromorphic function $M$ in $E^{*}$ is called a multiform solution of $\mathbb{C}$ in $E$ if

$$
\left(m_{i} \circ \lambda\right) M^{-1} \in \mathrm{H}^{0}\left(\lambda^{-1}\left(U_{i}\right), \bigcirc^{*}\right)
$$

for any $i \in I$. (5 has a multiform solution in $E$ if and only if " $(\rho(\mathbb{S}) \mid E)=0 . \quad \rho$ gives a homomorphism of the multiplicative group of all Cousin-II distributions in $D$ into $\mathrm{H}^{1}\left(D, \bigcirc^{*}\right)$. Let $\Im$ be a set of Cousin-II distributions in $D$. Let $D$ ₹ be the set of all subdomain $E$ of $D$ in which any distribution in $\mathfrak{F}$ has a multiform solution. Then we have $(D \Re, \subset)=\left(E_{\rho(\mathfrak{F})}, \subset\right)$. Hence we have the following Proposition.

Proposition 4. Let $\widetilde{F}$ be a set of Cousin-II distributions in a domain $D$ in a Stein manifold, $D_{\mathfrak{F}}$ be the set of all subdomains $E$ of $D$ in which any distribution in $\mathfrak{F}$ has a multiform solution and $\left(5\right.$ be a totally ordered subset of $\left(D_{\mathfrak{F}}, \subset\right)$. Then there exists a maximal element of $\left(D_{\mathfrak{\mho}}, \subset\right)$ which is an upper bound of $\mathfrak{}$.

Making use of Corollary of Lemma 8 we can also prove the following Proposition similarly.

Proposition 5. Let $\mathfrak{F}$ be a set of Cousin-I distributions in a domain $D$ in a Stein manifold, $D_{\mathfrak{\Im}}$ be the set of all subdomains $E$ of $D$ in which any distribution of $\mathfrak{F}$ has a solution and (5 be a totally ordered subset of $(D \Im, \subset)$. Then there exists a maximal element of $\left(D_{\Im}, \subset\right)$ which is an upper bound of $(\mathfrak{c}$.

\section{$\S 5$. Intersection of Cousin's domains}

In this paragraph we denote by $L$ a fixed, but arbitrary, abelian complex Lie group exclusively. A direct product $P$ of $n$ simply connected domains in a complex plane is called simply 
connected polycylinder in $C^{n}$. Of course we have $\mathrm{H}^{1}\left(P, \mathfrak{A}_{L}\right)=0$. In this paragraph we shall investigate intersections of domains $D$ with $\mathrm{H}^{1}\left(D, \mathfrak{A}_{L}\right)=0$ and extend Cartan-Behnke-Stein's theorem.

An open set $G$ in $C^{n}$ with $\mathrm{H}^{1}\left(G \cap P, \mathfrak{P}_{\boldsymbol{L}}\right)=0$ for any relatively compact and simply connected polycylinder $P$ in $C^{n}$ is called L-regular.

A domain $G$ in $C^{n}$ is said to be exhausted by L-regular domains $G_{p}$ if $G_{p}$ 's are $L$-regular domains in $C^{n}$ such that

$$
G_{p} \Subset G_{p+1}(p=1,2,3, \cdots) \text { and } G=\bigcup_{p=1}^{\infty} G_{p} .
$$

Lemma 10. Let $G$ be a domain in $C^{n}$ exhausted by L-regular domains $G_{p}$. Then ${ }^{*} \mathrm{H}^{1}\left(G, \mathfrak{A}_{L}\right)=0$. Moreover for any integers $1 \leqq m<n, \quad 1=s_{1}<s_{2}<\cdots<s_{n-m} \leqq n$ and for any complex numbers $c_{j}\left(j=s_{1}, s_{2}, \cdots s_{n-m}\right)$ the intersection $G \cap H$ of $G$ and $H=\left\{z=z_{1}, z_{2}\right.$, $\left.\left.\cdots, z_{n}\right) ; z_{j}=c_{j}\left(j=s_{1}, s_{2}, \cdots, s_{n-m}\right)\right\}$ satisfies ${ }^{*} \mathrm{H}^{1}\left(G \cap H, \mathfrak{A}_{L}\right)=0$.

Proof. Since $G_{p}$ is a relatively compact $L$-regular domain, we have $\mathbb{H}^{1}\left(G_{p}, \mathfrak{A}_{L}\right)=0$ for any $p$. From Lemma 9 we have ${ }^{\#} \mathrm{H}^{1}\left(G, \mathfrak{U}_{L}\right)=0$.

Next we shall prove ${ }^{\sharp} H^{1}\left(G \cap H, \mathfrak{U}_{\boldsymbol{L}}\right)=0$. We may assume that

$$
\begin{aligned}
H & =\left\{(z, w)=\left(z_{1}, z_{2}, \cdots, z_{m}, w_{1}, w_{2}, \cdots, w_{n-m}\right) ; w_{j}\right. \\
& =0(j=1,2, \cdots, n-m)\} .
\end{aligned}
$$

There exist $\varepsilon_{p}>0$ and $a_{p}>0$ such that

$$
\begin{gathered}
E_{p}=G_{p} \cap\left\{(z, w) ;\left|z_{j}\right|<a_{p},\left|w_{k}\right|<\varepsilon_{p}(j=1,2, \cdots, m ; k=1,2, \cdots, n-m)\right\} \\
\subset\left\{(z, w) ;\left|z_{j}\right|<a_{p},\left|w_{k}\right|<\varepsilon_{p},(z, 0) \in G \cap H,\right. \\
(j=1,2, \cdots, m ; k=1,2, \cdots, n-m)\}, \\
a_{p}<a_{p+1}(p \geqq 1) \text { and } a_{p} \rightarrow \infty(p \rightarrow \infty), \\
\varepsilon_{p}>\varepsilon_{p+1}(p \geqq 1) \text { and } \varepsilon_{p} \rightarrow 0(p \rightarrow \infty) .
\end{gathered}
$$

Since $G_{p}$ is $L$-regular, we have $\mathrm{H}^{1}\left(E_{p}, \mathfrak{A}_{L}\right)=0$ for any $p$. We put

$$
H_{p}=G_{p} \cap H \cap\left\{(z, 0) ;\left|z_{j}\right|<a_{p}(j=1,2, \cdots, m)\right\} .
$$


Then $G \cap H$ is the limit of a monotonously increasing sequence of open sets $H_{p}$ in $H$. Let $\mathfrak{B}=\left\{V_{s} ; s \in S\right\}$ be an open covering of $G \cap H$. We put $V_{s}^{p}=V_{s} \cap H_{p}$ for $s \in S$. Then $V_{p}=\left\{V_{s}^{p} ; s \in S\right\}$ is an open covering of $H_{p}$. We put

$$
U_{s}^{p}=E_{p} \cap\left\{(z, w) ;(z, 0) \in V_{s}\right\}
$$

for $s \in S$. Then $\mathfrak{U}_{p}=\left\{U_{s}^{p} ; s \in S\right\}$ is an open covering of $E_{p}$. Let $\left\{f_{s t}(z)\right\}$ be an element of $Z^{1}\left(\mathfrak{B}, \mathfrak{A}_{L}\right)$. We put

$$
F_{s t}^{p}(z, w)=f_{s t}(z)
$$

in $U_{s}^{p} \cap U_{t}^{p} \neq \phi . \quad$ Then $\left\{F_{s t}^{p}\right\} \in Z^{1}\left(\mathfrak{U}_{p}, \mathfrak{A}_{L}\right)=\mathbb{B}^{1}\left(\mathfrak{U}_{p}, \mathfrak{A}_{L}\right)$ from Lemma 1. There exists $F_{s}^{p} \in \mathbb{H}^{0}\left(U_{s}^{p}, \mathfrak{A}_{\boldsymbol{L}}\right)$ for any $s \in S$ such that

$$
F_{s t}^{p}=F_{s}^{p}\left(F_{t}^{p}\right)^{-1}
$$

in $U_{s}^{p} \cap U_{t}^{p} \neq \phi . \quad$ If we put

$$
f_{s}^{p}(z)=F_{s}^{p}(z, 0)
$$

in $V_{s}^{p}$ for any $s \in S$, then we have

$$
f_{s t}=f_{s}^{p}\left(f_{t}^{p}\right)^{-1}
$$

in $V_{s}^{p} \cap V_{t}^{p} \neq \phi$. Therefore the restriction of $\left\{f_{s t}\right\}$ in any $H_{p}$ is a coboundary of $\left\{f_{s}^{p}\right\} \in \mathrm{C}^{0}\left(\mathfrak{B}_{p}, \mathfrak{A}_{L}\right)$ for any $p$. From Lemma 1 $\left\{f_{s t} \circ \lambda\right\} \in \mathrm{B}^{1}\left(\lambda^{-1}(\mathfrak{B}), \mathfrak{A}_{L}\right),(G, \lambda)$ being the universal covering domain of $G$. Thus we have ${ }^{*} H^{1}\left(G \cap H, \mathfrak{A}_{L}\right)=0$.

From Proposition 2 and Lemma 10 we have

Proposition 6. A domain in $C^{n}$ exhausted by L-regular domains is a domain of holomorphy.

A boundary point $x^{0}$ of an open set $G$ in $R^{n}$ is called a continuous boundary point of $G$ if there exists a real-valued continuous function $g$ of variables $x_{1}, x_{2}, \cdots, \hat{x}_{9}, \cdots, x_{n}$ in a neighbounhood $V$ of $x^{0}$ such that

$$
\partial G \cap V=\left\{x=\left(x_{1}, x_{2}, \cdots, x_{n}\right) ; x_{j}=g\left(x_{1}, x_{2}, \cdots, \widehat{x}_{j}, \cdots, x_{n}\right), x \in V\right\}
$$

for some $j$. Moreover if $g$ is continuously differentiable in $U$, 
$x^{0}$ is called a smooth boundary point of $G$. An open set $G$ in $C^{p}$ is called pseudoconvex at a boundary point $x^{0}$ of $G$ if there exists an open neighbourhood $U$ of $x^{0}$ such that $G \cap U$ is an open set of holomorphy.

Proposition 7. An L-regular open set $G$ in $C^{n}$ is pseudoconvex at a continuous boundary point $z^{0}$ of $G$.

Proof. We put $z^{0}=\left(z_{1}^{0}, z_{2}^{0}, \cdots, z_{n}^{0}\right)$. We may assume that there exists $\varepsilon>0$ and a real-valued continuous function $g$ of variables $z_{1}, z_{2}, \cdots, z_{j-1}, y_{j}, z_{j+1}, \cdots, z_{n}$ in a neighbourhood $V=\left\{z=\left(z_{1}, z_{2}, \cdots\right.\right.$, $\left.\left.z_{n}\right) ;\left|z_{k}-z_{k}^{0}\right|<\varepsilon(k=1,2, \cdots, n)\right\}$ such that

$$
\partial G \bigcap V=\left\{z ; x_{j}=g\left(z_{1}, z_{2}, \cdots, z_{j-1}, y_{j}, z_{j+1}, \cdots, z_{n}\right), z \in V\right\}
$$

for some $j$ where $z_{j}=x_{j}+\sqrt{-1} y_{j}$. Then three cases (1), (2) and (3) may occur.

(1) $G \cap V=\left\{z ; x_{j}<g\left(z_{1}, z_{2}, \cdots, z_{j-1}, y_{j}, z_{j+1}, \cdots, z_{n}\right), z \in V\right\}$.

For $0 \leqq t<1$ we put

$$
V_{t}=\left\{z ;\left|z_{k}-z_{k}^{0}\right|<\frac{(1-t) \varepsilon}{2}(k=1,2, \cdots, m)\right\} .
$$

Then we have

$$
\left\{z ;\left(z_{1}, z_{2}, \cdots, z_{j-1}, z_{j}-\frac{t \varepsilon}{2}, z_{j+1}, \cdots, z_{n}\right) \in V_{t}\right\} \subset V
$$

for $0<t<1$. We put

$$
E_{t}=\left\{z ; x_{j}<g\left(z_{1}, z_{2}, \cdots, z_{j-1}, y_{j}, z_{j+1}, \cdots, z_{n}\right)-\frac{t \varepsilon}{2}, z \in V_{t}\right\} .
$$

Let $P$ be a relatively compact and simply connected polycylinder in $C_{n} . \quad E_{t} \cap P$ is mapped onto

$$
\begin{aligned}
& \left\{w ; u_{j}<g\left(w_{1}, w_{2}, \cdots, w_{j-1}, v_{j}, w_{j+1}, \cdots, w_{n}\right),\right. \\
& \left.\left(w_{1}, w_{2}, \cdots, w_{j-1}, w_{j}-\frac{t \varepsilon}{2}, w_{j+1}, \cdots, w_{n}\right) \in V_{t} \cap P\right\} \\
= & G \cap V \cap\left\{z ;\left(z_{1}, z_{2}, \cdots, z_{j-1}, z_{j}-\frac{t \varepsilon}{2}, z_{j+1}, \cdots, z_{n}\right) \in V_{t} \cap P\right\}
\end{aligned}
$$

by a biholomorphic mapping $w=\left(w_{1}, w_{2}, \cdots, w_{n}\right)=\gamma(z)$ defined by 
$w_{k}=z_{k}(k \neq j), w_{j}=z_{j}+t \varepsilon / 2$. Since $\gamma\left(E_{t} \cap P\right)$ is the intersection of $G$ and relatively compact and simply connected polycylinders, we have

$$
\mathrm{H}^{1}\left(E_{t} \cap P, \mathfrak{A}_{L}\right)=\mathrm{H}^{1}\left(\gamma\left(E_{t} \cap P\right), \mathfrak{A}_{L}\right)=0 .
$$

Therefore $E_{t}$ is an $L$-regular open set for $0 \leqq t<1$. Since $E_{0}$ is exhausted by $L$-regular domains $\left\{E_{t} ; 0<t<1\right\}, E_{0}=G \cap V_{0}$ is a domain of holomorphy from Proposition 1 . Hence $G$ is pseudoconvex at $z^{0}$.

(2) $G \cap V=\left\{z ; x_{j}>g\left(z_{1}, z_{2}, \cdots, z_{j-1}, y_{j}, z_{j+1}, \cdots, z_{n}\right), z \in V\right\}$.

In this case the situation is quite similar to the case (1).

(3) $G \cap V=\left\{z ; x_{j} \neq g\left(z_{1}, z_{2}, \cdots, z_{j-1}, y_{j}, z_{j+1}, \cdots, z_{n}\right), z \in V\right\}$.

Let

$$
G_{1}=\left\{z ; x_{j}<g\left(z_{1}, z_{2}, \cdots, z_{j-1}, y_{j}, z_{j+1}, \cdots, z_{n}\right), z \in V\right\}
$$

and

$$
G_{2}=\left\{z ; x_{j}>g\left(z_{1}, z_{2}, \cdots, z_{j-1}, y_{j}, z_{j+1}, \cdots, z_{n}\right), z \in V\right\} .
$$

From the cases (1) and (2) $G_{1}$ and $G_{2}$ are pseudoconvex at $z^{0}$. Hence $G \cap V=G_{1} \cup G_{2}$ is pseudoconvex at $z^{0}$.

Theorem 1. Let $L$ be an abelian complex Lie group. An L-regular domain $D$ in $C^{n}$ with a continuous boundary is a domain of holomorphy.

Proof. From Oka [16] and Proposition $7 D$ is a domain of holomorphy.

Theorem 2. Let $L$ be an abelian complex Lie group and $D$ be a domain with a smooth boundary in a Stein manifold $S$. If $\mathrm{H}^{1}\left(D \cap P, \mathfrak{N}_{L_{L}}\right)=0$ for any holomorphically convex subdomain $P$ of $S$ analytically contractible to each point of it, $D$ is holomorphically convex.

Proof. Let $x_{0}$ be a boundary point of $D$. There exists a biholomorphic mapping $\tau$ of an open neighbourhood $U^{\prime}$ of $x_{0}$ onto $Z^{\prime}=\left\{z ;\left|z_{1}\right|<2,\left|z_{2}\right|<2, \cdots,\left|z_{n}\right|<2\right\}$ and $\tau\left(x_{0}\right)=0$ where $n$ is the dimension of $S$. We put 


$$
Z=\left\{z ;\left|z_{1}\right|<1,\left|z_{2}\right|<1, \cdots,\left|z_{n}\right|<1\right\}
$$

and $U=\tau^{-1}(Z)$. Let $Q$ be any relatively compact and simply connected polycylinder in $C^{n}$. Then each connected component of $Q \cap Z$ is relatively compact and simply connected polycylinder in $C^{n}$. From Riemann's mapping theorem $Q \cap Z$ and $\tau^{-1}(Q \cap Z)$ are relatively compact Stein manifolds analytically contractible to each point of it. Since

$$
\mathrm{H}^{1}\left(Q \cap \tau(U \cap D), \mathfrak{A}_{L}\right)=\mathrm{H}^{1}\left(\tau^{-1}(Q \cap Z) \cap D, \mathfrak{A}_{L}\right)=0,
$$

$\tau(U \cap D)$ is $L$-regular and pseudoconvex at its smooth boundary point $x_{0}$ from Proposition 7. Therefore $D$ is pseudoconvex and is holomorphically convex from Docquier-Grauert [8].

Lemma 11. For any $p \geqq 1$ there exists homomorphism $\alpha$ of $\mathrm{GL}(1, C)$ in $\mathrm{GL}(p, C)$ and $\beta$ of $\mathrm{GL}(p, C)$ in $\mathrm{GL}(1, C)$ such that $\beta \circ \alpha$ is the identity of $\operatorname{GL}(1, C)$.

Proof. For $a \in \mathrm{GL}(1, C)$ we define the $p$ - $p$ matrix $\alpha(a)=\left(a_{i j}\right)$ by putting

$$
a_{11}=a, a_{i i}=1 \quad(i \geqq 2), \quad a_{i j}=0 \quad(i \neq j) .
$$

For $a \in \mathrm{GL}(p, C)$ we put $\beta(a)=\operatorname{det}(a)$. Then $\alpha$ and $\beta$ are desired homomorphisms.

Lemma 12. Let $L$ and $L^{\prime}$ be complex Lie groups with the following properties: There exist homomorphisms $\alpha$ of $L$ in $L^{\prime}$ and $\beta$ of $L^{\prime}$ in $L$ such that $\beta \circ \alpha$ is the identity of $L$. Then the canonical mapping $\mathrm{H}^{1}\left(X, \mathfrak{A}_{L}\right) \rightarrow \mathrm{H}^{1}\left(X, \mathfrak{A}_{L^{\prime}}\right)$ induced by $\alpha$ is injective for any complex space $X$.

Proof. Suppose that

$$
f_{\imath}^{-1}\left(\alpha \circ f_{i j}\right) f_{j}=\alpha \circ g_{i j}
$$

in $U_{i} \cap U_{j} \neq \phi$ for $\left\{f_{i j}\right\},\left\{g_{i j}\right\} \in \mathbb{Z}^{1}\left(\mathfrak{U}, \mathfrak{A}_{L}\right)$ and $\left\{f_{i}\right\} \in \mathbb{C}^{0}\left(\mathfrak{U}, \mathfrak{A}_{L^{\prime}}\right)$ where $\mathfrak{U}=\left\{U_{i} ; i \in I\right\}$ is an open covering of $X$. Then $\left\{\beta \circ f_{i}\right\} \in \mathrm{C}^{\circ}\left(\mathfrak{U}, \mathfrak{H}_{L}\right)$ satisfies

$$
\left(\beta \circ f_{i}\right)^{-1} f_{i j}\left(\beta \circ f_{j}\right)=g_{i j}
$$


in $U_{i} \cap U_{j} \neq \phi$.

From Lemmas 11 and 12 we have the following Theorem.

Theorem 3. For any positive integer p Propositions 1,2,6 and 7 Theorems 1 and 2 are valid if we replace $L$ by $\mathrm{GL}(p, C)$.

$\S 6$. The sheaf $\mathfrak{M}$ of all germs of meromorphic functions

In the previous paper [10] we remarked that $\mathrm{H}^{1}(D, \mathfrak{M})=0$ for any 1-dimensional Stein manifold $D$, that is, for any noncompact Riemann surface $D$ where $\mathfrak{M}$ is the sheaf of all germs of meromorphic functions. But we have the following Proposition for a domain in $C^{n}(n \geqq 2)$.

Proposition 8. Let $D$ be a domain in $C^{n}$ ( $\left.n \geqq 2\right)$. Then $\mathrm{H}^{1}(D, \mathfrak{M}) \neq 0$.

Proof. Suppose that $\mathrm{H}^{1}(D, \mathfrak{M})=0$. For the sake of brevity of notations any point of $C^{n}$ is denoted by $x=\left(z, w, w_{3}, \cdots, w_{n}\right)$. Then there exist complex numbers $a$ and $b$ with $0 \neq a \neq b \neq 0$ such that

$$
D_{1}=D-\{x ; z=a\} \varsubsetneqq D, D_{2}=D-\{x ; z=b\} \varsubsetneqq D .
$$

From Lemma 1 we have $H^{1}(\mathfrak{U}, \mathfrak{M})=0$ for the open covering $\mathfrak{U}=\left\{D_{1}, D_{2}\right\}$ of $D$. We put

$$
m^{\prime}=\frac{1}{w-\exp \left((z-a)^{-1}(z-b)^{-1}\right)} \in \mathrm{H}^{0}\left(D_{1} \cap D_{2}, \mathfrak{M}\right) .
$$

There exists $m_{1}^{\prime} \in \mathrm{H}^{0}\left(D_{1}, \mathfrak{M}\right)$ and $m_{2}^{\prime} \in \mathrm{H}^{0}\left(D_{2}, \mathfrak{M}\right)$ such that

$$
m^{\prime}=m_{1}^{\prime}-m_{2}^{\prime}
$$

in $D_{1} \cap D_{2}$. The pole surfaces $A^{\prime}, A_{1}^{\prime}$ and $A_{2}^{\prime}$ of $m^{\prime}, m_{1}^{\prime}$ and $m_{2}^{\prime}$ are, respectively, analytic sets in $D_{1} \cap D_{2}, D_{1}$ and $D_{2}$. There holds

$$
A^{\prime} \subset\left(A_{1}^{\prime} \cup A_{2}^{\prime}\right) \cap D_{1} \cap D_{2}
$$

Since $A^{\prime}$ is irreducible at each point of it, we have $A^{\prime} \subset A_{1} \cap D_{1}$ $\cap D_{2}$ or $A^{\prime} \subset A_{2} \cap D_{1} \cap D_{2}$. Without loss of generality we may suppose that $A^{\prime} \subset A_{2}^{\prime} \cap D_{1} \cap D_{2}$. Since $D_{1} \varsubsetneqq D$, there exists $x^{0}=$ 
$\left(a, a_{2}, a_{3}, \cdots, a_{n}\right) \in D$ for our $a$. There exists $\delta>0$ such that

$$
\begin{gathered}
U=\left\{x=\left(z, w, w_{3}, \cdots, w_{n}\right) ;|z-a|<\delta,\left|w-a_{2}\right|<\delta,\left|w_{3}-a_{3}\right|<\delta,\right. \\
\left.\cdots,\left|w_{n}-a_{n}\right|<\delta\right\} \subset D .
\end{gathered}
$$

There exists an integer $p \geqq 0$ such that the germ of the pole surfaces of

$$
m_{2}=(z-a)^{p} m_{2}^{\prime} \in \mathrm{H}^{0}\left(D_{2}, \mathfrak{M}\right)
$$

at $x^{0}$ does not contain that of $\{x ; z=a\}$ at $x^{0}$. We put

$$
m=(z-a)^{p} m^{\prime} \in \mathrm{H}^{0}\left(D_{1} \cap D_{2}, \mathfrak{M}\right) \text {. }
$$

The pole surfaces of $A$ and $A_{2}$ of $m$ and $m_{2}$ are, respectively, analytic sets in $D_{1} \cap D_{2}$ and $D_{2}$ satisfying

$$
A \subset A_{2} \cap D_{1} \cap D_{2} \text {. }
$$

Let $x=\left(a, b_{2}, b_{3}, \cdots, b_{n}\right)$ be any point of $U \cap\{x ; z=a\}$ with $b_{2} \neq 0$. There exists an integer $q_{0}^{\prime}>0$ such that

$$
\left|\frac{4}{\log b_{2}+i\left(\arg b_{2}+2 q \pi\right)}\right|<|a-b|^{2}
$$

for $q \geqq q_{0}^{\prime}$. We put $x^{q}=\left(a^{(q)}, b_{2}, \cdots, b_{n}\right)\left(q \geqq q_{0}^{\prime}\right)$ for

$$
a^{(q)}=\frac{a+b+\sqrt{(a-b)^{2}+\frac{4}{\log b_{2}+i\left(\arg b_{2}+2 q \pi\right)}}}{2}
$$

where the function $\sqrt{z}$ is defined in $\left\{z ;\left|z-(a-b)^{2}\right|<|a-b|^{2}\right\}$ so that $\sqrt{(a-b)^{2}}=a-b$. There exists $q_{0}\left(>q_{0}^{\prime}\right)$ such that

$$
x^{q} \in U
$$

for $q>q_{0}^{\prime}$. Then we have

$$
x^{q} \in A\left(q>q_{0}\right), x^{q} \rightarrow x(q \rightarrow \infty) .
$$

Since $A \subset A_{2}, A_{2}$ is an analytic set in $D_{2}$ and $D_{2}$ contains $x^{(\varphi)}$ $\left(q<q_{0}\right)$ and $x$, we have $x \in A_{2}$. This means that

$$
U \cap\{x ; z=a\} \subset A_{2} .
$$

But this contradicts to the fact that the germ of $A_{2}$ at $x^{0}$ does 
not contain that of $\{x ; z=a\}$ at $x^{0}$. Thus we have proved that $\mathrm{H}^{1}(D, \mathfrak{M}) \neq 0$.

\section{REFERENCES}

[1] Behnke, H., Généralisation du théorème de Runge pour les fonctions multiformes des variables complexes. Coll. sur les fonct. des plus. var. Bruxelles (1953).

[2] Behnke, H., und K. Stein, Analytische Funktionen mehrer Veränderlichen zur vorgegebenen Null- und Pollstellenflächen. Jber. Deut. Math. Verein. 47 (1937), 177-192.

[3] - Entwicklung analytischer Funktionen auf Riemannschen Flächen. Math. Ann. 120 (1948), 430-461.

[4] Bochner, S., and W. T. Martin, Several complex variables. Princeton Univ. Press (1948).

[5] Cartan, H., Les problèmes de Poincaré et de Cousin pour les fonctions de plusieurs variables complexes. C. R. Paris 199 (1934), 1284-1287.

[6] — Sur les premières problèmes de Cousin. C. R. Paris 207 (1938), 558-560.

[7] Cartan, H., et al., Théorie des fonctions de plusieurs variables: Sem. de Cartan, Ecole Norm., Sup. (1951-52).

[8] Docquier, F., und H. Grauert, Levisches Problem und Rungescher Satz fur Teilgebiete Steinscher Mannigfaltigkeit. Math. Ann. 140 (1960), 94-123.

[9] Eilenberg, S., and N. Steenrod, Foundations of algebraic topology. Princeton Univ. Press.

[10] Kajiwara, J., On an application of L. Ehrenpreis's method to ordinary differential equations. Kōdai. Math. Sem. Rep. 15 (1963), 94-105.

[11] - On the limit of a monotonous sequence of Cousin's domains. J. Math. Soc. Japan 17 (1965), 36-46.

[12] - Some characterizations of Stein manifold through the notion of locally regular boundary points. Kōdai Math. Sem. Rep. 16 (1964), 36-46.

[13] - Note on a Cousin-II domain over C2. Ibid. 17 (1965), 44-47.

[14] Relations between domains of holomorphy and multiple Cousin's problems. Ibid., 261-272.

[15] Oka, K., Sur les fonctions analytiques de plusieurs variables: II Domaines d'holomorphie. J. Sci. Hiroshima Univ. 7 (1937), 115-130.

[16] — : IX Domaines finis sans point critique intérieur. Jap. J. Math. 23 (1953), 97-155.

[17] Stein, K., Überlagerungen holomorph-vollständiger komplexer Räume. Arch. Math. 7 (1956), 354-361.

[18] Thullen, P., Sur les deusième problème de Cousin. C. R. Paris 200 (1935), 720-721. 